\title{
Junctional Bradycardia as Early Sign of Digoxin Toxicity in a Premature Infant with Congestive Heart Failure due to a Left to Right Shunt
}

\author{
${ }^{1}$ Department of Pediatrics, University of Texas Medical Branch, \\ University Boulevard, Galveston, Texas \\ 2 Department of Pediatric Cardiology and MFM, University of Texas \\ Medical Branch, University Boulevard, Galveston, Texas \\ ${ }^{3}$ Department of Neonatology, University of Texas Medical Branch, \\ University Boulevard, Galveston, Texas
}

Soham Dasgupta, MD ${ }^{1}$ Ashraf M. Aly, MD, PhD² Sunil K. Jain, MD³

\author{
Address for correspondence Sunil K. Jain, MD, Department of \\ Neonatology, University of Texas Medical Branch, 301 University \\ Boulevard, Galveston, TX-77550 (e-mail: skjain@utmb.edu).
}

Am J Perinatol Rep 2016;6:e96-e98.

\begin{abstract}
Introduction Congestive heart failure due to left to right cardiac shunt is usually managed medically with diuretics, angiotensin converting enzyme inhibitors, and, in some cases, with the addition of digoxin.

Case We report a 31-week gestation premature male infant who did not respond to such treatment and developed hyperaldosteronism and severe hypokalemia secondary to activation of the renin angiotensin aldosterone system. The hypokalemia was not responsive to intravenous $\mathrm{KCL}$ supplementation and induced digoxin toxicity despite a relatively normal digoxin level. The earliest signs of digoxin toxicity in the patient were junctional rhythm and bradycardia. The discontinuation of digoxin and the administration of digoxin specific immunoglobulin fragments (Fab) reversed those changes. The

Keywords

- digoxin

- junctional rhythm

- hyperaldosteronism

- hypokalemia

- congestive heart failure addition of spironolactone (an aldosterone antagonist) had a dramatic effect, resulting in clinical improvement of the patient coupled with normalization of Q4 serum and urine electrolytes.

Conclusion Serum Digoxin level alone may fail as an independent guide in the diagnosis of digoxin toxicity when hypokalemia is present. In premature infants with congestive heart failure and hypokalemia, addition of an aldosterone antagonist should be considered.
\end{abstract}

Congestive heart failure (CHF) is a clinical syndrome resulting from inappropriate tissue perfusion and is a major cause of morbidity and mortality in children. ${ }^{1}$ In neonates with congenital cardiac lesions with left to right shunt like ventricular septal defect (VSD), the effective systemic perfusion could be compromised by the decreased systemic blood flow and the decreased vascular tone due to the immature autonomic nervous system. ${ }^{2}$ The decreased effective systemic blood flow leads to decreased renal perfusion and increased renin production from the kidney, which activates the renin angiotensin aldosterone system

received

July 29, 2015

accepted after revision

October 6, 2015

published online

November 20, 2015
(RAAS). This leads to hyperaldosteronism, which may cause fluid retention and electrolyte imbalance especially hypokalemia. ${ }^{3}$ CHF due to left to right cardiac shunt is usually treated with diuretics (preload reduction), angiotensin converting enzyme inhibitors (afterload reduction), and in some cases, with the addition of digoxin (primarily for its cholinergic effect).

We describe a premature male infant with a large left to right shunt who presented with junctional bradycardia as an early sign of digoxin toxicity due to activation of the RAAS.

Copyright $@ 2016$ by Thieme Medical Publishers, Inc., 333 Seventh Avenue, New York, NY 10001, USA. Tel: +1(212) 584-4662.
License terms

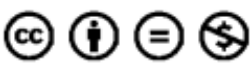

$10.1055 / \mathrm{s}-0035-1567858$ ISSN 2157-6998. 


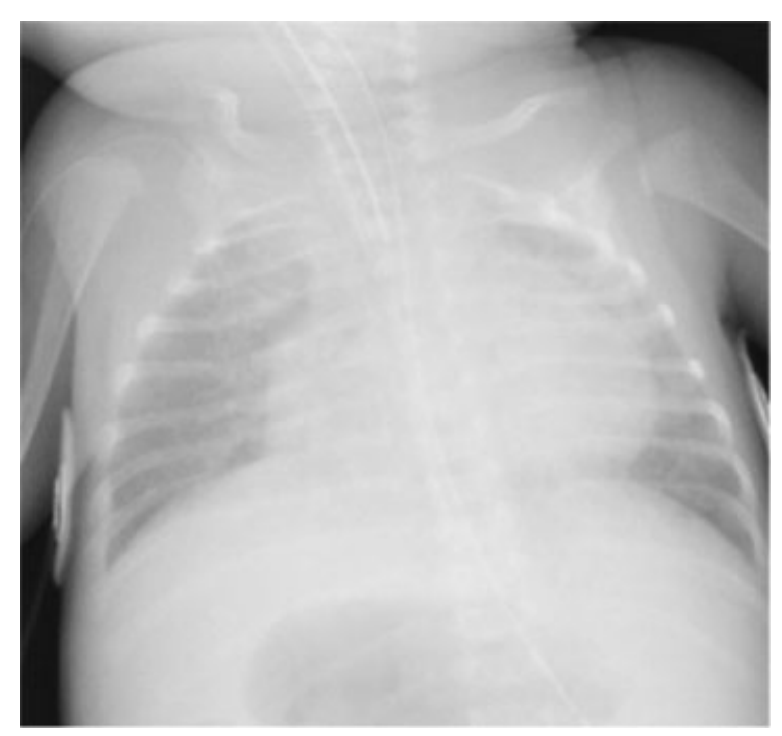

Fig. 1 Chest X-ray showing cardiomegaly and pulmonary edema.

\section{Case Report}

A premature male infant was born at 31 weeks' gestation that was complicated by oligohydramnios. Physical examination was remarkable for retrognathia, high arched palate, undescended testes, and hypotonia. On day 3 of life, a holosystolic murmur was appreciated and an echocardiogram showed a moderate-size membranous VSD, a moderate-size patent ductus arteriosus, and a small-size secundum atrial septal defect. He had normal serum electrolytes on day 5 of life. At 2 weeks of age, he developed progressive shortness of breath in the form of tachypnea and intercostal retractions. The mean arterial pressure remained in the normal range (50-60 mm $\mathrm{Hg}$ ). A chest X-ray showed cardiomegaly and pulmonary edema suggestive of CHF (-Fig. 1). At that time, he was treated with furosemide and captopril. Two days later and because of poor clinical response, digoxin was added. Digitalis-like product levels were checked prior to the administration of digoxin $(0.5 \mathrm{ng} / \mathrm{mL})$. One day after intravenous (IV) digitalization was complete, the urine output decreased $(<1 \mathrm{~mL} / \mathrm{kg} / \mathrm{h}$ ) and blood urea nitrogen increased to $27 \mathrm{mg} / \mathrm{dL}$. Basic metabolic panel showed severe hypokalemia $(1.9 \mathrm{mmol} / \mathrm{L})$ and hypernatremia $(149 \mathrm{mmol} / \mathrm{L})$. Urinary potassium was $111.8 \mathrm{mmol} / \mathrm{L}$ (normal is $<10 \mathrm{mmol} / \mathrm{L}$ ) and urinary sodium $<5 \mathrm{mmol} / \mathrm{L}$ (normal $20-40 \mathrm{mmol} / \mathrm{L}$ ). The hypokalemia did not respond to IV KCL supplementation. These results were suggestive of hyperaldosteronism (serum aldosterone was $547.5 \mathrm{ng} / \mathrm{dl}$; normal 7-99 ng/dL). The patient developed episodes of bradycardia with the heart rate dropping to the $40 \mathrm{~s}$ (beats/min) as noted on the heart monitor. The electrocardiogram (EKG) showed junctional rhythm with ventricular rates of 70 beats/min. The serum digoxin level was $2.20 \mathrm{ng} / \mathrm{mL}$ (normal 1.1-1.7 ng/mL). Digoxin was immediately discontinued, digoxin specific immunoglobulin fragments (Fab) was administered and spironolactone, an aldosterone antagonist, was added. Within 12 hours of changing the treatment plan, the EKG normalized to sinus rhythm with a heart rate of 120 beats/min. The serum and urinary sodium and potassium levels along with serum aldosterone levels normalized within 4 days. Clinically, the patient improved significantly and was extubated to be maintained on continuous positive airway pressure.

Due to dysmorphic facial features, karyotyping and microarray analysis were performed and showed partial trisomy 8 mosaicism. The routine newborn screen was normal. The $\mathrm{CHF}$ was medically managed for 1 month before the patient rapidly deteriorated and developed severe pulmonary hypertension requiring mechanical ventilation again. A repeat echocardiogram showed severe pulmonary hypertension with right to left shunt at the level of the VSD. He did not respond to maximum support and eventually expired at 6 weeks of age.

\section{Discussion}

The sodium potassium $(\mathrm{Na} / \mathrm{K})$ ATPase pump exists in three isoforms in humans. ${ }^{4}$ The primary effect of digoxin is to inhibit membrane bound $\alpha$ subunits of the $\mathrm{Na} / \mathrm{K}$ ATPase pump mainly but not exclusively in the human myocardium. ${ }^{4}$ This inhibition promotes sodium-calcium exchange, which increases the intracellular calcium concentration, ${ }^{4}$ resulting in an increase in the force of myocardial contraction-positive inotropic effect. ${ }^{4}$ However, digoxin has additional neurohormonal effects. ${ }^{5}$ Studies have shown that such effects may also be secondary to inhibition of the $\mathrm{Na} / \mathrm{K}$ ATPase pump. ${ }^{6}$ Neuro-hormonal effects of digoxin include attenuation of carotid sinus baroreceptor discharge sensitivity leading to decreased sympathetic nervous system activation, central vagal stimulation, and a direct sympatho-inhibitory effect on the myocardium. ${ }^{5}$ This increases the vagal tone to the heart leading to decreased conduction velocity across the atrioventricular (AV) node and increased refractoriness of the AV node to impulses originating from the sinoatrial node (SA) node. ${ }^{7}$ At therapeutic doses, digoxin does not appear to increase the risk of cardiac arrhythmias. ${ }^{8}$ However, toxic levels of digoxin are known to cause cardiac arrhythmias and also other central nervous system and gastrointestinal abnormalities. Digoxin toxicity leads to a high degree of AV block by its effect of increasing vagal tone to the heart. As a result, very few impulses originating from the SA node are conducted to the ventricles. In such cases, a slower pacemaker takes over the function of the SA node leading to junctional, sub-junctional, or ventricular arrhythmias. ${ }^{7}$ Digoxin toxicity has been documented to cause junctional rhythm with slow ventricular rate of $<100$ beats/min in infants. ${ }^{7}$

Digoxin toxicity is not only dose-dependent but may also result from decreased clearance of digoxin in renal insufficiency. Serum levels of digoxin $>2 \mathrm{ng} / \mathrm{mL}^{8}$ are associated with toxic effects with the recommended digoxin serum levels being from 1.1 to $1.7 \mathrm{ng} / \mathrm{mL}^{8}$ Digoxin toxicity may also be accentuated by electrolyte imbalance (hypokalemia) secondary to activation of RAAS. The pharmacological properties of the isoforms of the $\mathrm{Na} / \mathrm{K}$ ATPase pump explain the role of hypokalemia in digoxin toxicity. ${ }^{4}$ The $\alpha$ subunit of the NA/K ATPase pump binds digoxin, sodium, potassium, 
and ATP. ${ }^{4}$ The $\alpha 1$ and 3 isoforms have high half-times for the dissociation of bound digoxin of 80 and 30 minutes, respectively. ${ }^{4}$ At normal serum potassium levels, these isoforms exhibit lower sensitivities to digoxin. Potassium thus exerts a protective effect ${ }^{4}$ and prevents excess digoxin from binding to the $\mathrm{Na} / \mathrm{K}$ ATPase pump. This may be explained by potassium competing with digoxin for myocardial $\mathrm{Na} / \mathrm{K}$ ATPase receptor binding sites. However, in the presence of hypokalemia, more digoxin is able to bind to the $\alpha 1$ and 3 isoforms of the $\mathrm{Na} / \mathrm{K}$ ATPase pump. Once bound, the long dissociation half-times lead to prolonged $\mathrm{Na} / \mathrm{K}$ ATPase pump inhibition. This leads to a decrease in the conduction velocity of impulses across the $\mathrm{AV}$ node and may lead to the development of an escape junctional rhythm. Thus, toxicity may be seen at normal serum digoxin levels in the presence of hypokalemia. ${ }^{9}$

In our patient, the activation of the RAAS secondary to CHF led to severe hypokalemia. The digoxin level was $2.2 \mathrm{ng} / \mathrm{mL}(0.5$ $\mathrm{ng} / \mathrm{mL}$ was digitalis-like products). Therefore, the actual serum digoxin level was $1.7 \mathrm{ng} / \mathrm{mL}$. This is within the recommended ranges for serum digoxin levels. However, the hypokalemia caused by RAAS activation accentuated the digoxin toxicity even at that therapeutic serum digoxin level. ${ }^{10}$

Digoxin toxicity was confirmed by the presence of junctional rhythm on $\mathrm{EKG}^{11}$ and the reversal to normal sinus rhythm after discontinuing digoxin and the administration of Fab. Fab forms a complex with digoxin; hence digoxin is not available to the myocytes. Fab also combines with digoxin bound to $\mathrm{Na} / \mathrm{K}$ ATPase pumps and removes digoxin from these pumps. ${ }^{4}$ The Fab-digoxin complex is then excreted in the urine. $^{12}$ In the management of CHF with electrolyte imbalance such as hypokalemia, which could be secondary to RAAS activation, the addition of an aldosterone antagonist and a potassium sparing diuretic such as spironolactone should be considered. This may prevent the activation of the RAAS and the occurrence of severe hypokalemia, thus preventing the occurrence of life-threatening arrhythmias in a patient receiving digoxin for the management of $\mathrm{CHF}$.

\section{Conclusion}

In premature infants with $\mathrm{CHF}$, digoxin toxicity should be suspected in the presence of hypokalemia, which could be secondary to RAAS activation. We suggest careful electrolyte monitoring and the judicious use of digoxin in such patients. Serum digoxin level alone may fail as an independent guide to the diagnosis of digoxin toxicity in the presence of hypokalemia. $^{10}$ The hypokalemia may not respond well to IV KCL supplementation. We thus recommend monitoring for digoxin toxicity by performing interval EKGs ${ }^{11}$ and monitoring of serum potassium levels along with serum digoxin levels. In those cases, the addition of aldosterone antagonists like spironolactone should be considered.

\section{References}

1 Borlaug BA, Paulus WJ. Heart failure with preserved ejection fraction: pathophysiology, diagnosis, and treatment. Eur Heart J 2011;32(6):670-679

2 Friedman WF. The intrinsic physiologic properties of the developing heart. Prog Cardiovasc Dis 1972;15(1):87-111

3 Tait JF, Little B, Tait SA, Flood C, Bougas J. Splanchnic extraction and clearance of aldosterone in subjects with minimal and marked cardiac dysfunction. J Clin Endocrinol Metab 1965; $25: 219-228$

4 Lelievre LG, Lechat P. Mechanisms, manifestations and management of digoxin toxicity. Heart Metab 2007;35:9-11

5 Gheorghiade M, Adams KF Jr, Colucci WS. Digoxin in the management of cardiovascular disorders. Circulation 2004;109(24): 2959-2964

6 Wang W, Chen JS, Zucker IH. Carotid sinus baroreceptor sensitivity in experimental heart failure. Circulation 1990;81(6): 1959-1966

7 Smith TW, Antman EM, Friedman PL, Blatt CM, Marsh JD. Digitalis glycosides: mechanisms and manifestations of toxicity. Part I. Prog Cardiovasc Dis 1984;26(5):413-458

8 Park MK. Use of digoxin in infants and children, with specific emphasis on dosage. J Pediatr 1986;108(6):871-877

9 Bhatia SJ. Digitalis toxicity-turning over a new leaf? West J Med 1986;145(1):74-82

10 Sundar S, Burma DP, Vaish SK. Digoxin toxicity and electrolytes: a correlative study. Acta Cardiol 1983;38(2):115-123

11 Ma G, Brady WJ, Pollack M, Chan TC. Electrocardiographic manifestations: digitalis toxicity. J Emerg Med 2001;20(2): 145-152

12 Felicilda-Reynaldo RF. Cardiac glycosides, digoxin toxicity, and the antidote. Medsurg Nurs 2013;22(4):258-261 